\title{
Incidence and risk factors of parastomal hernia after radical cystectomy and ileal conduit diversion: a systematic review and meta-analysis
}

\author{
Dechao Feng ${ }^{1,2 \#}$, Zhenghao Wang ${ }^{1 \#}$, Yubo Yang ${ }^{1 \wedge}$, Dengxiong $\mathrm{Li}^{1} \wedge$, Wuran $\mathrm{Wei}^{1 \wedge}, \mathrm{Li} \mathrm{Li}^{2,3}$ \\ ${ }^{1}$ Department of Urology, Institute of Urology, West China Hospital, Sichuan University, Chengdu, China; ${ }^{2}$ Key Laboratory of Birth Defects and \\ Related Diseases of Women and Children (Sichuan University), Ministry of Education, Chengdu, China; ${ }^{3}$ Department of Pediatrics, West China \\ Second University Hospital, Sichuan University, Chengdu, China \\ Contributions: (I) Conception and design: D Feng; (II) Administrative support: L Li; (III) Provision of study materials or patients: D Feng, Z Wang, Y \\ Yang; (IV) Collection and assembly of data: D Li, W Wei, Y Yang; (V) Data analysis and interpretation: D Feng, Z Wang; (VI) Manuscript drafting: \\ All authors; (VII) Final approval of manuscript: All authors. \\ "These authors contributed equally to this work. \\ Correspondence to: Li Li, PhD. Department of Pediatrics, West China Second University Hospital, Sichuan University, No. 20, Section 3, Renmin \\ Nan Lu, Chengdu 610041, China. Email: liliwch@126.com.
}

Background: Our aim is to report the incidence and risk factors of parastomal hernia (PH) after radical cystectomy (RC) and ileal conduit (IC) diversion with a cumulative analysis.

Methods: Various databases, including PubMed, the Cochrane Library, Embase and Web of Science, were retrieved electronically and manually to identify eligible studies from inception to August 20, 2020. Two reviewers independently searched the above databases and selected the studies using prespecified standardized criteria. The Newcastle-Ottawa Scale (NOS) was used to assess the risk of bias in the included studies, and the data was completed by STATA version 14.2.

Results: Fifteen studies were included in the final analysis. A pooled analysis of eight studies representing 1,878 patients reported the incidence of overall radiographic $\mathrm{PH}$ was $23 \%$ (95\% CI: 17-29\%). The 1-year $\mathrm{PH}$ incidence rate and 2-year incidence rate of RC and IC were 14\% (95\% CI: 6-22\%) and 26\% (95\% CI: 14-38\%), respectively. A pooled analysis of nine studies reported the incidence of clinically evident $\mathrm{PH}$ was $15 \%$ (95\% CI: 10-19\%). PH-related symptoms were reported in six studies, and the pooled result was $29 \%$ (95\% CI: 24-33\%), and a pooled analysis of ten studies showed that 20\% (95\% CI: 11-28\%) of patients required surgical repair. However, it's noteworthy that among symptomatic $\mathrm{PH}$ patients undergoing surgical repair, the pooled analysis of five studies showed that up to $26 \%$ (95\% CI: $16-36 \%$ ) of patients suffered $\mathrm{PH}$ recurrence. The most frequent risk factor was body mass index (BMI). Patients with BMI $\geq 22.9 \mathrm{~kg} / \mathrm{m}^{2}$ experienced 2.92-fold higher risk of $\mathrm{PH}$ than their counterparts [hazard ratio (HR): 2.92; 95\% CI: 1.65-5.19]. Conclusions: Our findings indicated that the $\mathrm{PH}$ incidence rate after $\mathrm{RC}$ and $\mathrm{IC}$ was significantly higher in radiographic evaluation than that of clinical examination, and the recurrence of repairment is considerable for patients requiring reconstruction.

Keywords: Parastomal hernia (PH); ileal conduit diversion (IC diversion); radical cystectomy (RC); bladder cancer

Submitted Nov 30, 2020. Accepted for publication Jan 22, 2021.

doi: $10.21037 /$ tcr-20-3349

View this article at: http://dx.doi.org/10.21037/tcr-20-3349

\footnotetext{
^ ORCID: Dechao Feng, 0000-0002-8267-9920; Yubo Yang, 0000-0002-0189-3256; Dengxiong Li, 0000-0002-1943-6758; Wuran Wei, 0000-0002-2133-6043.
} 


\section{Introduction}

Bladder cancer is one of the most pernicious urologic caners globally, with an estimated 549,000 new cases and 200,000 death each year (1). It is universally acknowledged that BC not only poses a great threat on patients' existence and quality of life, but also increases drastically the economic burden of the national health care system (2). Radical cystectomy (RC) and ileal conduit (IC) diversion has currently been regarded as the mainstream treatment of muscle-invasive and high-risk non-muscle-invasive bladder cancer (3). However, this approach is usually associated with noteworthy complications and higher mortality concerning the complexity of the operation involving urinary and intestinal systems (4).

It is conceivable that the urinary diversion (UD) contributes to great risk of postoperative morbidity and complications other than cystectomy itself $(4,5)$. Among the long-term complications reported, parastomal hernia $(\mathrm{PH})$ is a frequent sequela resulting in cosmetic and functional concerns, and surgical repair is required in some cases, such as intestinal incarceration and strangulation $(6,7)$. Concerning the incongruous incidence and risk factors, and deficiency of effective treatments, we determine to report the finding of this review with a cumulative analysis of incidence and risk factors of $\mathrm{PH}$ in patients undergoing $\mathrm{RC}$ and IC, and explore the potentially effective treatments for this medical problem. We present the following article in accordance with the PRISMA reporting checklist (available at http://dx.doi.org/10.21037/tcr-20-3349).

\section{Methods}

\section{Study selection}

In accordance with the Preferred Reporting Items for Systematic Review and Meta-analyses guidelines (8), various databases including PubMed, the Cochrane Library, Embase and Web of Science were systematically searched through electronic retrieval from inception to August 20, 2020 without language limitations. The Medical Subject Heading $(\mathrm{MeSH})$ terms related to this article included "Hernia" and "Cystectomy", and the search strategy used in PubMed was as follows: ((Cystectomy[Title/ Abstract]) OR (Cystectomies[Title/Abstract])) AND (((Hernia[Title/Abstract]) OR (Hernias[Title/Abstract])) OR (Enterocele[Title/Abstract])). The specific search strategy is showed in Appendix 1. We also artificially retrieved the reference lists of relevant reviews and articles to expand the search. Two independent authors identified the potential studies for full-text evaluation based on screening titles and abstracts. Subsequently, articles that met the inclusion criteria were included in the final analysis. Data were extracted by two independent authors, and any disagreement was resolved by consensus or a third party. By formulating a table previously, we extracted the following data: (I) the first author name and publication year; (II) details of the study design; (III) the characteristics of the included patients, and (IV) data associated with outcomes of interest.

\section{Selection criteria}

We used the following PICOS method to identify the qualification of included studies: (I) patients $(\mathrm{P})$ : patients undergoing RC and IC; sample size greater than 90; radiographically, $\mathrm{PH}$ was defined as evidence of the protrusion of abdominal contents through the abdominal wall defect created by forming the stoma. When present, $\mathrm{PH}$ grade was recorded using a previously published classification system of type 1-hernia sac that contains the prolapsed bowel loop forming the stoma, type 2-contains abdominal fat or omentum herniating through the abdominal wall defect created by the stoma and type 3 -contains herniated loops of bowel other than that forming the stoma (9). Clinically, $\mathrm{PH}$ is defined as any protrusion in the vicinity of the urostoma with the patient straining in a supine and an erect position; (II) intervention (I): patients without any precaution for $\mathrm{PH}$ formation; (III) comparison; not applicable; (IV); outcomes (O): any study reporting incidence or risk factors of $\mathrm{PH}$ after $\mathrm{RC}$ and IC was collected. The main outcomes were the $\mathrm{PH}$ incidence rates of overall radiographic imaging and clinical examination; The secondary outcomes were the PH incidence of overall symptom, surgical repair and recurrence of reconstruction, and risk factors associated with PH. (V) Study design (S): full-text studies published with English. For articles with potentially overlapping population, only the largest report was included, unless they reported different outcomes of interest. Besides, exclusion criteria were as follows: (I) any study which did not satisfy the inclusion criteria; (II) single-case report, meeting abstracts, review or meta-analysis; (III) data not available. Figure 1 sketches the PRISMA flowchart of this meta-analysis.

\section{Quality assessment}

The methodological quality of included studies was assessed 


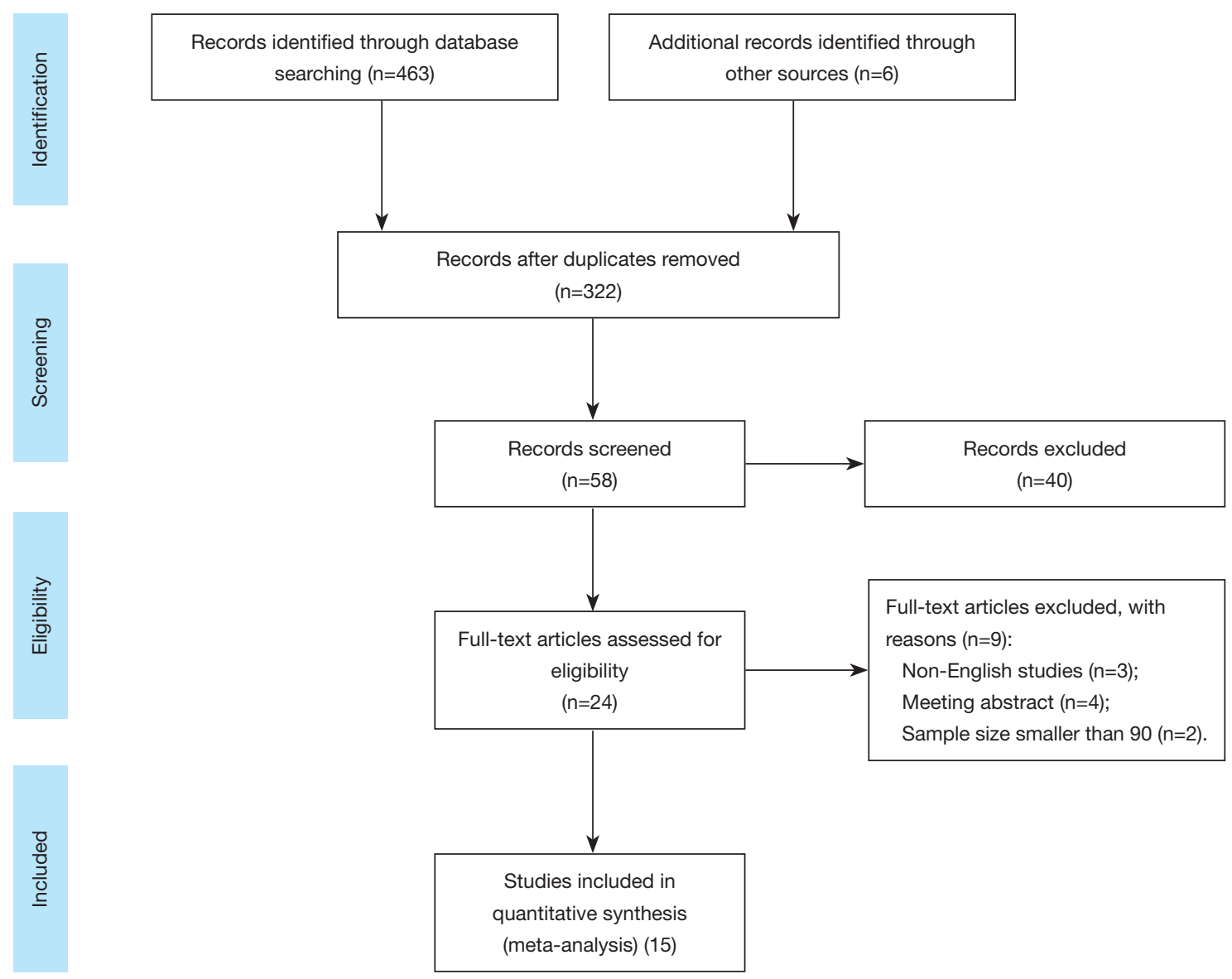

Figure 1 The PRISMA flowchart.

by two independent reviewers using the Newcastle-Ottawa Scale (NOS) (10). The NOS applied a 'star system' to evaluate the quality of study from three perspectives: the selection of the studies, the comparability of studies, and the assessment of outcome. If seven or more stars were received, the study was considered as to be high-quality. Furthermore, two reviewers independently rated the level of evidence of the included articles through the Oxford Centre for Evidence-Based Medicine criteria (11). This scale graded studies from strongest (level 1) to weakest (level 5) strength of evidence according to study design and data quality.

\section{Statistical analysis}

The $\mathrm{Q}$ and $\mathrm{I}^{2}$ tests were calculated to assess the heterogeneity among studies. Pooled rates (PRs) or hazard ratio (HR) and corresponding 95\% CIs were calculated through random-effect model unless the between-study heterogeneity was endurable $\left(\mathrm{P}>0.1\right.$ or $\left.\mathrm{I}^{2} \leq 50 \%\right)$. We quantified the asymmetry of funnel plots through the Egger's test and Begg's test to evaluate the underlying publication bias. A $\mathrm{P}$ value of less than 0.1 was regarded as significant. In addition, we conducted a sensitivity analysis through single factor analysis to evaluate the robustness of the pooled effects. For all statistical analyses, two-sided $\mathrm{P}<0.05$ was considered statistically significant. Data from each study were pooled where possible, otherwise narrative review was conducted. This meta-analysis was accomplished through STATA version 14.2.

\section{Results}

\section{Literature search results}

After removing duplicates, screening titles and abstracts, and evaluating full texts, $15(9,12-25)$ of 469 studies containing 4,252 patients were included to evaluate the incidence of 
$\mathrm{PH}$ after RC and IC. The studied population came from USA $(9,12,14,15,18,20,24,25)$, China (22), Switzerland (23), Denmark (13), Japan (16,17), Spain (19), and Sweden (21). The vast majority of articles were reported retrospectively, and the span of median time to diagnosis of $\mathrm{PH}$ was from 11 to 28.8 months. Table 1 showed the characteristics of included studies in this meta-analysis.

\section{Incidence}

A total of eight studies $(9,12,15,16,18-21)$ representing 1,878 patients reported the incidence of overall radiographic $\mathrm{PH}$, and the rates ranged from $10 \%$ to $35 \%$. As integrated, PH developed in 23\% (95\% CI: 17-29\%; Figure 2) of patients who underwent RC and IC. The between-study heterogeneity was obvious $\left(\mathrm{P}<0.001, \mathrm{I}^{2}=90.8 \%\right.$; Figure 2$)$. A pooled analysis of three studies $(9,18,21)$ showed that the $\mathrm{PH}$ incidence rate of open RC (ORC) and IC was 27\% (95\% CI: 19-36\%; Figure 2). The 1-year PH incidence rate and 2 -year incidence rate of RC and IC were 14\% (95\% CI: 6-22\%; Figure 2) and 26\% (95\% CI: 14-38\%; Figure 2), respectively. Similarly, patients undergoing ORC and IC had a higher risk of 2-year radiographic PH (PR: 37\%; 95\% CI: 14-59\%; Figure 2). The incidence of clinically evident $\mathrm{PH}$ after $\mathrm{RC}$ and IC was provided in nine studies including 2,433 patients $(9,13,14,17,21-25)$, and the synthesized result was $15 \%$ (95\% CI: $10-$ $19 \%$; Figure 2). The heterogeneity among studies was also detected $\left(\mathrm{P}<0.001, \mathrm{I}^{2}=90.2 \%\right.$; Figure 2$)$. Only two studies $(9,12)$ reported the incidence of type 1 $\mathrm{PH}$ and type $2 \mathrm{PH}$, and the corresponding PRs were 1\% (95\% CI: 0-2\%; Figure 2) and 16\% (95\% CI: 4-29\%; Figure 2), respectively. A total of three studies $(9,12,21)$ provided the incidence of type $3 \mathrm{PH}$, and the pooled result was $10 \%$ (95\% CI: $8-12 \%$; Figure 2). Only Donahue et al. (9) reported the results of progression to a higher type of hernia. They observed that $80 \%(1 / 5)$ of initial type 1 hernias progressed to type 3 while $33 \%$ (30/90) of initial type 2 hernias progressed to type 3 . In addition, Hussein et al. (12) reported that $32 \%$ patients developed $\mathrm{PH}$ after robot-assisted RC (RARC) and IC at 3 years.

PH-related symptoms, such as pain, bowel incarceration, appliance difficulties, discomfort, or PH-induced leakage, were reported in six studies $(9,12,14,16,19,20)$, and the incidence rate ranged from $15 \%$ to $44 \%$. The pooled results indicated that 29\% (95\% CI: $24-33 \%$; Figure 3) of $\mathrm{PH}$ patients were at risk of presenting symptoms without significant between-study heterogeneity
$\left(\mathrm{P}=0.302, \mathrm{I}^{2}=17.2 \%\right.$; Figure 3$)$. A pooled analysis of ten studies $(9,12,14-21)$ including 455 symptomatic patients showed that 20\% (95\% CI: $11-28 \%$; Figure 3) of patients who underwent RC and IC required surgical repair with significant heterogeneity among studies $(\mathrm{P}<0.001$, $\mathrm{I}^{2}=82.8 \%$; Figure 3). Besides, the surgical repair rate of symptomatic patients after ORC and IC was provided in three studies $(9,18,21)$, and the synthesized result was $7 \%$ (95\% CI: 3-11\%; Figure 3). We did not observe an evident between-study heterogeneity $\left(\mathrm{P}=0.163, \mathrm{I}^{2}=44.9 \%\right.$; Figure 3$)$. However, it's noteworthy that among symptomatic $\mathrm{PH}$ patients undergoing surgical repair, the pooled analysis of five studies $(9,12,14,15,19)$ showed that up to $26 \%(95 \%$ CI: 16-36\%; Figure 3) of patients suffered PH recurrence.

\section{Risk factors}

A wide array of independent risk factors was reported. A pooled analysis of two studies $(12,16)$ showed that patients with fascial defect size $24 \mathrm{~mm}$ or greater experienced 6.80fold higher risk of $\mathrm{PH}$ than their counterparts (HR: 6.80; 95\% CI: 3.54-13.06; Figure 3). A pooled analysis of two studies $(9,21)$ showed that per increased unit of body mass index (BMI) was associated with the increasing risk of $\mathrm{PH}$ (HR: 1.05; 92\% CI: 1.05-1.11). Furthermore, patients with $\mathrm{BMI} \geq 22.9 \mathrm{~kg} / \mathrm{m}^{2}$ had 2.17 times higher risk of $\mathrm{PH}$ than their counterparts (HR: 2.17; 95\% CI: 0.81-5.80) (16). Other significantly reported risk factors included female (HR: 2.25; 95\% CI 1.58-3.21) (9), preoperative albumin (HR: 0.43 ; 95\% CI: 0.25-0.75) (9), longer operative time [odds ratio (OR): 1.25 ; 95\% CI: 1.06-1.46] (12), postoperative eGFR less than $60 \mathrm{~mL}$ per minute (OR: 2.17, 95\% CI: 1.23-3.90) (12), prior exploratory laparotomy (HR: 1.98; 95\% CI: 1.97-3.36) (15), prior tobacco use (OR: 0.23; 95\% CI: 0.09-0.63) (20), anterior fixation (OR: 2.3; 95\% CI: $1.03-5.14)(24)$, prophylactic mesh (OR: 0.49; $95 \%$ CI: $0.26-0.95)(21)$ and surgery in one of the hospitals (OR: 3.34; 95\% CI: 1.39-8.06) (21).

\section{Publication bias and sensitivity analysis}

We employed the Egger's test and Begg's test to quantify the asymmetry of funnel plots, and $\mathrm{P}$ value greater than 0.1 meant no obvious publication bias. The results of Egger's test and Begg's test related to the main outcomes were provided in Table S1. Generally speaking, we did not detect noticeable publication bias. We evaluated the impact of a single study on the combined effect size through 


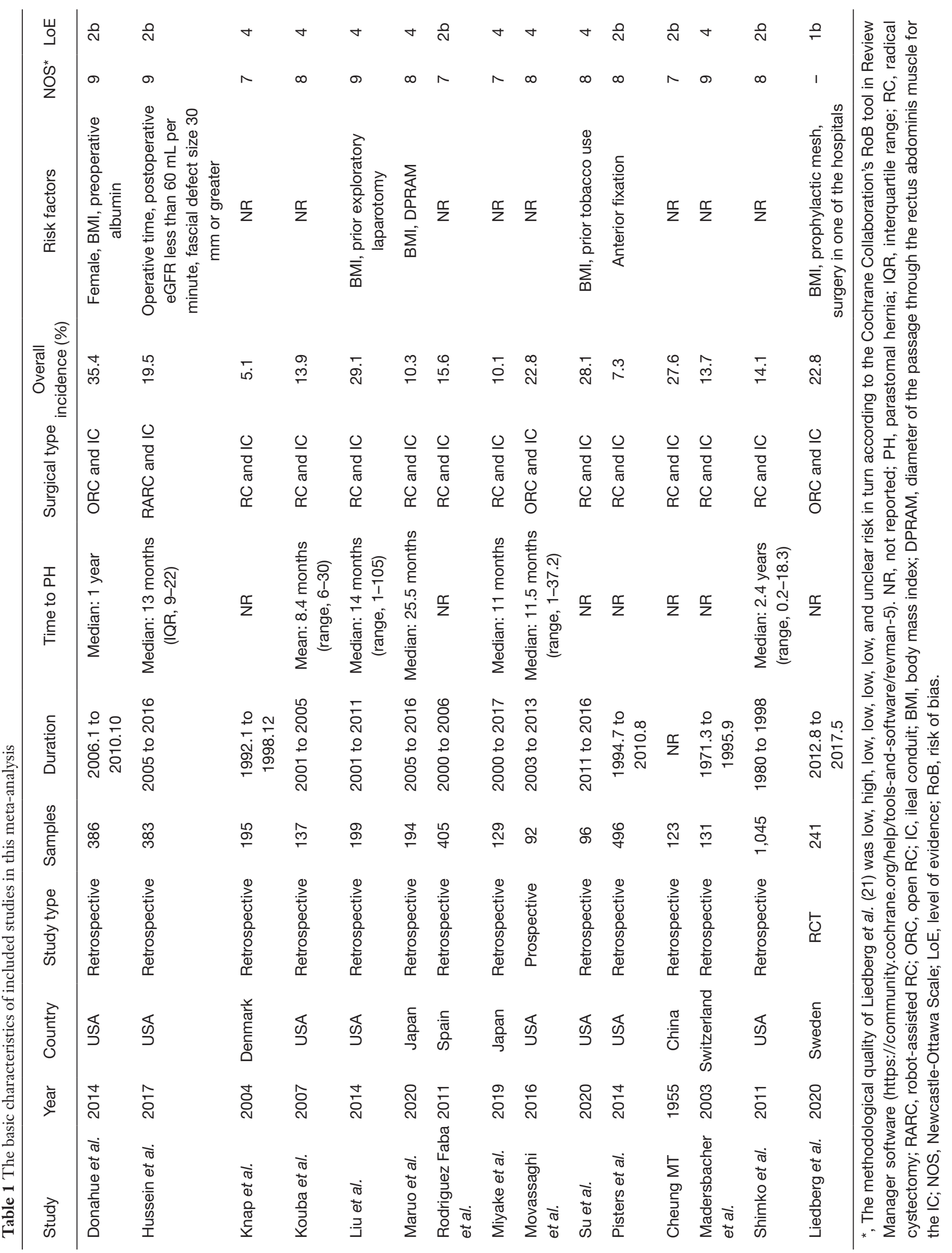


Overall radiographic $\mathrm{PH}$

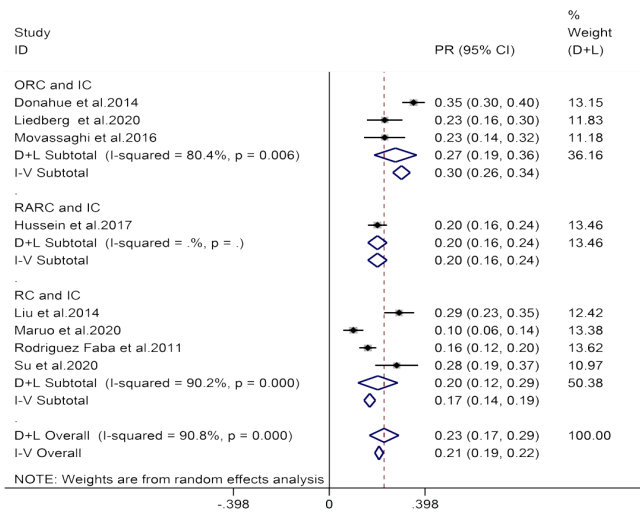

2-yr radiographic $\mathrm{PH}$

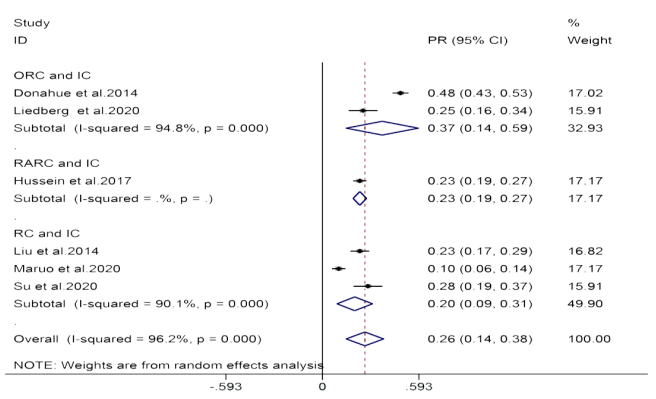

Type 1 overall radiographic $\mathrm{PH}$

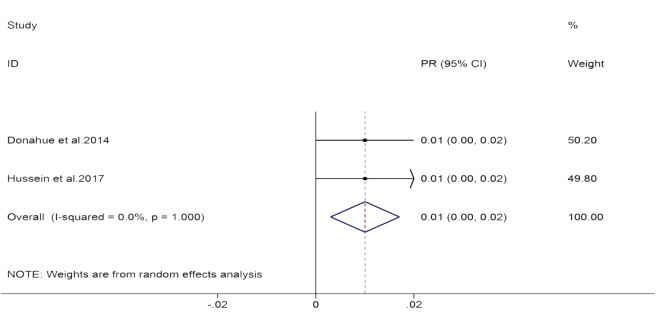

Type 3 overall radiographic $\mathrm{PH}$

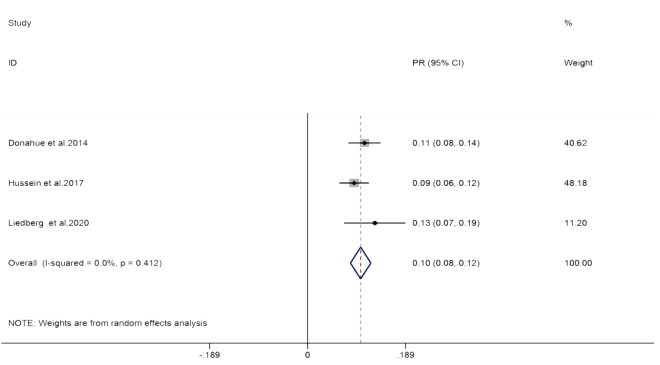

1-yr radiographic $\mathrm{PH}$

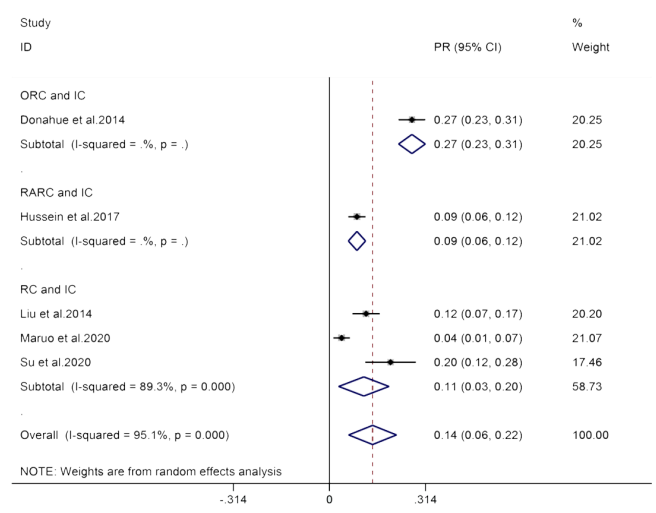

Clinically evident $\mathrm{PH}$

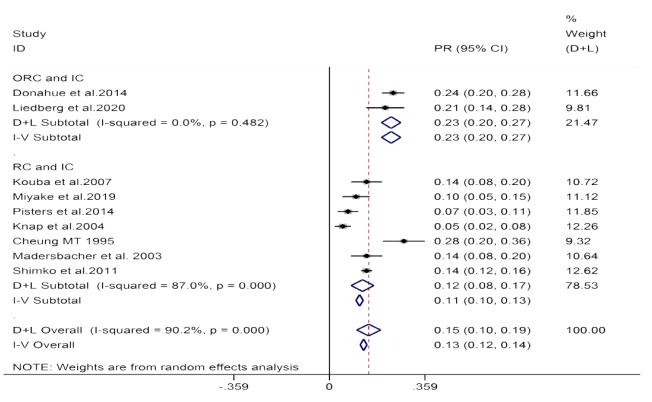

Type 2 overall radiographic $\mathrm{PH}$

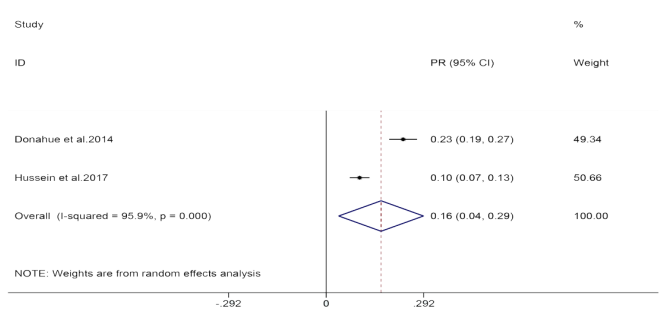

Figure 2 The pooled results of the primary outcomes in this study. PH, parastomal hernia; PR, pooled rate; RC, radical cystectomy; ORC, open RC; RARC, robot-assisted RC; IC, ileal conduit. 
Overall symptoms-related incidence

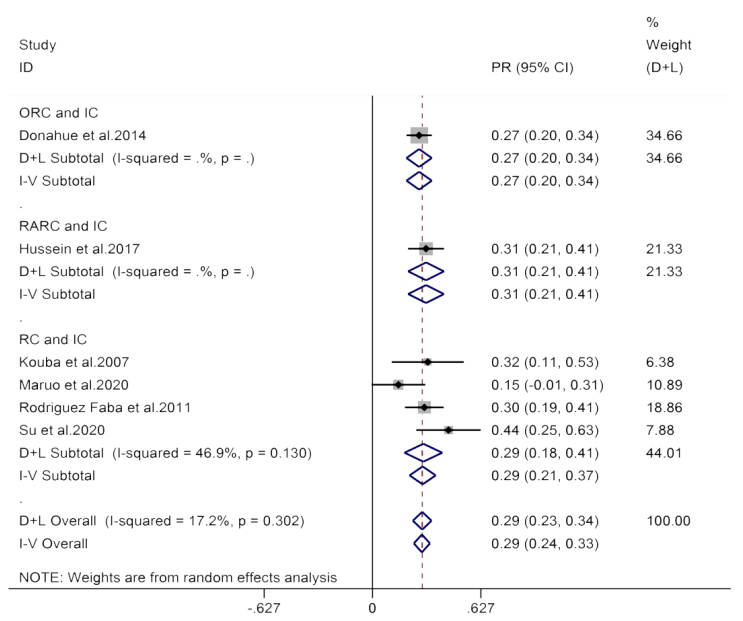

Recurrence of surgical repair

\section{Surgical repair}

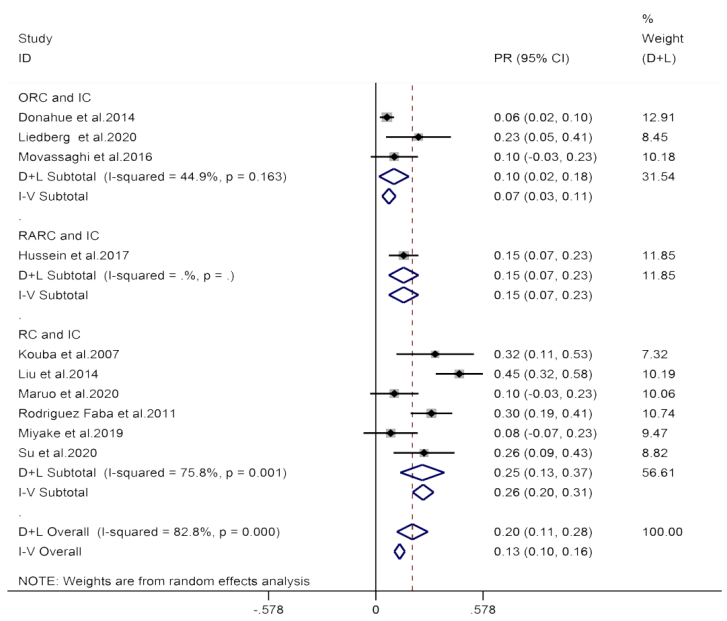

Fascial defect size $\geq 24 \mathrm{~mm}$

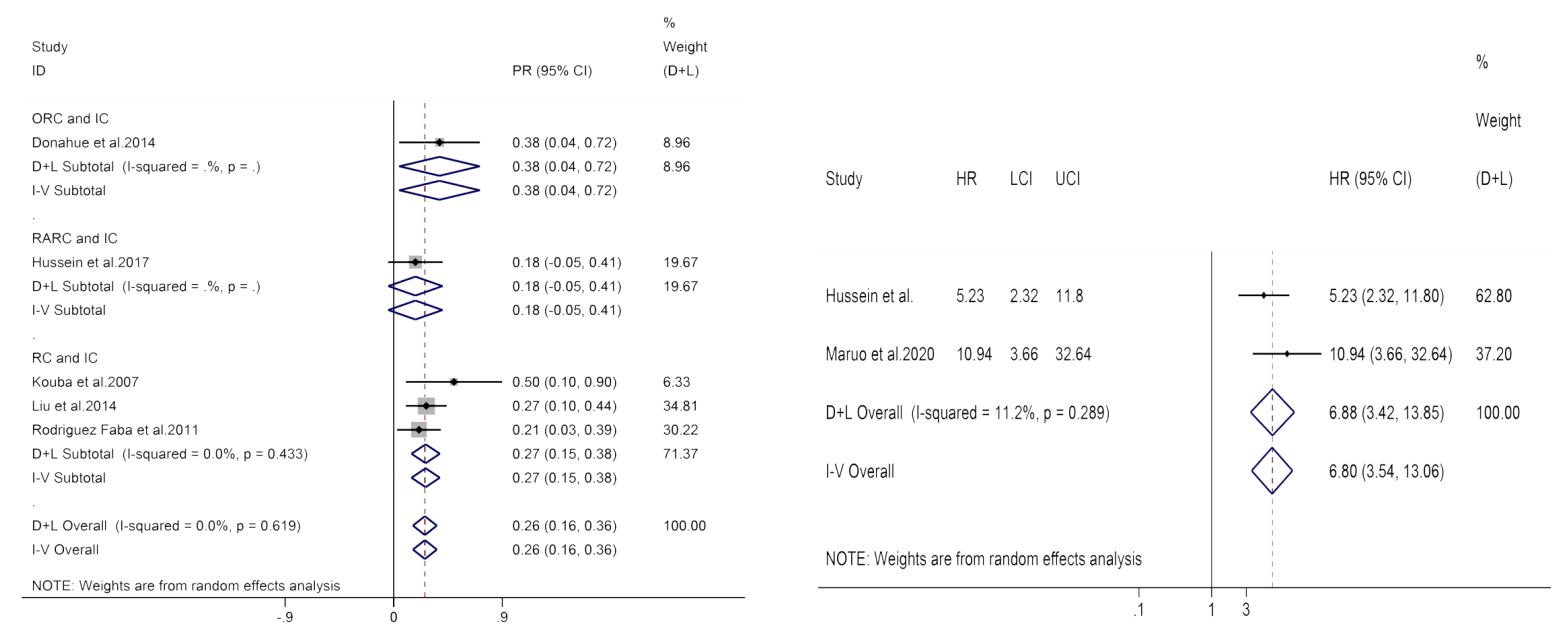

Figure 3 The pooled results of the secondary outcomes in this study. PR, pooled rate; RC, radical cystectomy; ORC, open RC; RARC, robot-assisted RC; IC, ileal conduit.

excluding each study in sequence, and the results of the sensitivity analysis indicated that the pooled effects were robust (Figure S1).

\section{Discussion}

Perioperative outcomes and complications are the primary two aspects that patients are most concerned about. Postoperative complications are inevitable for complex procedures such as RC. As is known to all, ORC with extended pelvic lymph node dissection has been gold standard for patients with muscle-invasive and highrisk non-muscle-invasive bladder cancer for decades (3). However, conspicuous complications and higher mortality leave much to be desired (4). Although our previous studies indicated that minimally invasive approaches, scilicet RARC and laparoscopic RC (LRC), presented improved perioperative outcomes and comparable pathological and oncologic outcomes at the cost of longer operative time when compared to ORC $(26,27)$, on no account can we ignore the fact that many of the perioperative complications following $\mathrm{RC}$ might derive from the reconstructive part 
of the procedure, and overwhelming majority of studies included in our meta-analysis were insufficient information of stomal-related complication, such as $\mathrm{PH}$ whose incidence was not low (PR: 23\%; 95\% CI: 17-29\%).

$\mathrm{RC}$ is generally accompanied by the UD, which is closely related to the patient's long-term quality of life. Since it was introduced by Seiffert and popularized by Bricker (23), the IC has been the gold standard for those with incontinent UD and has still been the primary choice for patients with contraindications to continent UD for over seven decades due to its simplicity and practicability $(16,17)$. Extensively used as IC is, stoma-related complications are rarely reported in spite of relatively high incidence of $25 \%$ to $60 \%$ (14). $\mathrm{PH}$ is a relatively common complication with specific to IC. In our study, the synthesized rate was $23 \%$, and the $\mathrm{PH}$ incidence seemed to be associated with length of follow up. The $\mathrm{PH}$ incidence rate at 1 and 2 years were $14 \%$ and $26 \%$, respectively. Besides, the detection rate of radiographic evaluation is higher than that of clinical examination ( $23 \%$ vs. $15 \%)$. The reason was that clinical evaluation is enslaved to variation in terms of whether it is patient reported $v s$. documented on physical examination, and whether examination was performed with the patient supine or upright and with or without the Valsalva maneuver $(6,12)$. On the other hand, radiological images are more objective, less prone to variation due to body habitus and not subject to bias while it also enables consideration of other aspects, including rectus muscle defects and abdominal wall thickness, which may contribute to $\mathrm{PH}(6,12)$.

In most cases, $\mathrm{PH}$ is symptomless and incidentally diagnosed during cancer surveillance. In our cumulative analysis, a total of $29 \%$ of $\mathrm{PH}$ patients suffered from symptoms. Symptoms related to $\mathrm{PH}$ included pain, discomfort, bowel incarceration. Moreover, if the hernia increases in size, distortion of the abdominal wall might obstruct the application of the stoma bag, leading to urine leakage. Additionally, our study indicated that a total of $20 \%$ of symptomatic patients with $\mathrm{PH}$ required surgical correction, but the recurrence of surgical repair was as great as $26 \%$. There are currently conflicting results of risk factors associated with $\mathrm{PH}$. Obesity is the most frequently reported factor among them. Based on our clinical experience, diameter of the passage through the rectus abdominis muscle for the IC (DPRAM), prophylactic mesh, and surgery in one of the hospitals are the most potential factors in addition to obesity. Several corrective techniques have been reported to repair $\mathrm{PH}$, including translocation of the stoma (19), anterior fixation (24), and mesh usage $(7,21,28-30)$. Prophylactic mesh placement has been demonstrated safe and effective in reducing $\mathrm{PH}$ rate for patients undergoing elective colorectal operation (31). However, there is a paucity of data alluding to the use of mesh in prevention or repairment of $\mathrm{PH}$. Considering that a $\mathrm{PH}$ constitutes a significant clinical problem for the individual patient, it pays to be further investigated the role of a prophylactic mesh.

Our study did have the following limitations. First of all, there were inherent limitations in the included studies of this meta-analysis. The retrospective study may lead to selection bias, such as Berkson bias and Neyman bias. Secondly, the broad heterogeneity in study populations, designs and definitions of outcome measures. Finally, different follow-up duration detected among the included studies also affected the incidence rates, and we were unable to evaluate the long-term incidence rate of $\mathrm{PH}$.

\section{Conclusions}

Our findings indicated that the $\mathrm{PH}$ incidence rate of radiographic evaluation and clinical examination were $23 \%$ and $15 \%$, respectively. A total of $29 \%$ of $\mathrm{PH}$ patients suffered from symptoms, and a total of $20 \%$ of symptomatic patients with $\mathrm{PH}$ required surgical correction. However, the recurrence of surgical repair was as great as $26 \%$. Given that a $\mathrm{PH}$ constitutes a noticeable clinical problem for the individual patient, it pays to be further investigated the $\mathrm{PH}-$ related risk factors and the role of a prophylactic mesh.

\section{Acknowledgments}

Funding: This work was supported by Department of Science and Technology of Sichuan Province (2020YFH0099) and the National Natural Science Foundation of China (No. 81370272, 30901621/C1705).

\section{Footnote}

Reporting Checklist: The authors have completed the PRISMA reporting checklist. Available at http://dx.doi. org/10.21037/tcr-20-3349

Conflicts of Interest: All authors have completed the ICMJE uniform disclosure form (available at http://dx.doi. org/10.21037/tcr-20-3349). The authors have no conflicts of interest to declare. 
Ethical Statement: The authors are accountable for all aspects of the work in ensuring that questions related to the accuracy or integrity of any part of the work are appropriately investigated and resolved.

Open Access Statement: This is an Open Access article distributed in accordance with the Creative Commons Attribution-NonCommercial-NoDerivs 4.0 International License (CC BY-NC-ND 4.0), which permits the noncommercial replication and distribution of the article with the strict proviso that no changes or edits are made and the original work is properly cited (including links to both the formal publication through the relevant DOI and the license). See: https://creativecommons.org/licenses/by-nc-nd/4.0/.

\section{References}

1. Bray F, Ferlay J, Soerjomataram I, et al. Global cancer statistics 2018: GLOBOCAN estimates of incidence and mortality worldwide for 36 cancers in 185 countries. CA Cancer J Clin 2018;68:394-424.

2. Leal J, Luengo-Fernandez R, Sullivan R, et al. Economic burden of bladder cancer across the European Union. Eur Urol 2016;69:438-47.

3. Witjes JA, Bruins HM, Cathomas R, et al. EAU guidelines on muscle-invasive and metastatic bladder cancer. The Netherlands: European Association of Urology; 2020; Available online: https://uroweb.org/guideline/bladdercancer-muscle-invasive-and-metastatic/

4. Feng D, Tang $Y$, Yang $Y$, et al. Intracorporeal versus extracorporeal urinary diversion after robotic-assisted radical cystectomy: evidence from a systematic review and pooled analysis of observational studies. Minerva Urol Nefrol 2020;72:519-30.

5. Patel HR, Santos PB, de Oliveira MC, et al. Is roboticassisted radical cystectomy (RARC) with intracorporeal diversion becoming the new gold standard of care? World J Urol 2016;34:25-32.

6. Donahue TF, Bochner BH. Parastomal hernias after radical cystectomy and ileal conduit diversion. Investig Clin Urol 2016;57:240-8.

7. Donahue TF, Cha EK, Bochner BH. Rationale and early experience with prophylactic placement of mesh to prevent parastomal hernia formation after ileal conduit urinary diversion and cystectomy for bladder cancer. Curr Urol Rep 2016;17:9.

8. Moher D, Liberati A, Tetzlaff J, et al. Preferred reporting items for systematic reviews and meta-analyses: the
PRISMA statement. PLoS Med 2009;6:e1000097.

9. Donahue TF, Bochner BH, Sfakianos JP, et al. Risk factors for the development of parastomal hernia after radical cystectomy. J Urol 2014;191:1708-13.

10. Wells GA, Shea B, O'Connell D, et al. The NewcastleOttawa Scale (NOS) for assessing the quality if nonrandomized studies in meta-analyses. Ottawa Hosp Res Inst. Available online: http://www.ohri.ca/programs/ clinical_epidemiology/oxford.asp (Accessed August 22, 2020).

11. Centre for Evidence-Based. Oxford Centre for EvidenceBased Medicine: levels of evidence (March 2009). 2009. Available online: https://www.cebm.ox.ac.uk/resources/ levels-of-evidence/oxford-centre-for-evidence-basedmedicine-levels-of-evidence-march-2009

12. Hussein AA, Ahmed YE, May P, et al. Natural history and predictors of parastomal hernia after robot-assisted radical cystectomy and ileal conduit urinary diversion. J Urol 2018;199:766-73.

13. Knap MM, Lundbeck F, Overgaard J. Early and late treatment-related morbidity following radical cystectomy. Scand J Urol Nephrol 2004;38:153-60.

14. Kouba E, Sands M, Lentz A, et al. Incidence and risk factors of stomal complications in patients undergoing cystectomy with ileal conduit urinary diversion for bladder cancer. J Urol 2007;178:950-4.

15. Liu NW, Hackney JT, Gellhaus PT, et al. Incidence and risk factors of parastomal hernia in patients undergoing radical cystectomy and ileal conduit diversion. J Urol 2014;191:1313-8.

16. Maruo K, Tanaka T, Shindo T, et al. Incidence and risk factors of parastomal hernia after ileal conduit diversion in Japanese population. Int J Clin Oncol 2020;25:1830-4.

17. Miyake M, Owari T, Tomizawa $M$, et al. Long-term changes in renal function, blood electrolyte levels, and nutritional indices after radical cystectomy and ileal conduit in patients with bladder cancer. Urol J 2019;16:145-51.

18. Movassaghi K, Shah SH, Cai J, et al. Incisional and parastomal hernia following radical cystectomy and urinary diversion: the University of Southern California experience. J Urol 2016;196:777-81.

19. Rodriguez Faba O, Rosales A, Breda A, et al. Simplified technique for parastomal hernia repair after radical cystectomy and ileal conduit creation. Urology 2011;77:1491-4.

20. Su JS, Hoy NY, Fafaj A, et al. The European Hernia Society classification applied to the rare cases of parastomal 
hernia after ileal conduit urinary diversion: a retrospective cohort of 96 patients. Hernia 2021;25:125-31.

21. Liedberg F, Kollberg P, Allerbo M, et al. Preventing parastomal hernia after ileal conduit by the use of a prophylactic mesh: a randomised study. Eur Urol 2020;78:757-63.

22. Cheung MT. Complications of an abdominal stoma: an analysis of 322 stomas. Aust N Z J Surg 1995;65:808-11.

23. Madersbacher S, Schmidt J, Eberle JM, et al. Long-term outcome of ileal conduit diversion. J Urol 2003;169:985-90.

24. Pisters AL, Kamat AM, Wei W, et al. Anterior fascial fixation does not reduce the parastomal hernia rate after radical cystectomy and ileal conduit. Urology 2014;83:1427-31.

25. Shimko MS, Tollefson MK, Umbreit EC, et al. Longterm complications of conduit urinary diversion. J Urol 2011;185:562-7.

26. Feng D, Liu S, Tang Y, et al. Comparison of perioperative and oncologic outcomes between robot-assisted and laparoscopic radical cystectomy for bladder cancer: a systematic review and updated meta-analysis. Int Urol

Cite this article as: Feng D, Wang Z, Yang Y, Li D, Wei W, $\mathrm{Li}$ L. Incidence and risk factors of parastomal hernia after radical cystectomy and ileal conduit diversion: a systematic review and meta-analysis. Transl Cancer Res 2021;10(3):1389-1398. doi: $10.21037 /$ tcr-20-3349
Nephrol 2020;52:1243-54.

27. Feng D, Li A, Hu X, et al. Comparative effectiveness of open, laparoscopic and robot-assisted radical cystectomy for bladder cancer: a systematic review and network metaanalysis. Minerva Urol Nefrol 2020;72:251-64.

28. Tully KH, Roghmann F, Pastor J, et al. Parastomal hernia repair with 3-D mesh implants after radical cystectomy and ileal conduit urinary diversion - a single-center experience using a purpose made alloplastic mesh implant. Urology 2019;131:245-9.

29. Tenzel PL, Williams ZF, McCarthy RA, et al. Prophylactic mesh used in ileal conduit formation following radical cystectomy: a retrospective cohort. Hernia 2018;22:781-4.

30. Styrke J, Johansson M, Granåsen G, et al. Parastomal hernia after ileal conduit with a prophylactic mesh: a 10 year consecutive case series. Scand J Urol 2015;49:308-12.

31. Pianka F, Probst P, Keller AV, et al. Prophylactic mesh placement for the PREvention of paraSTOmal hernias: the PRESTO systematic review and meta-analysis. PLoS One 2017;12:e0171548. 
Supplementary

\section{Appendix 1}

\section{Search strategy}

PubMed

Hernia (MeSH term)

Hernias

Enterocele

Cystectomy (MeSH term)

Cystectomies

((Cystectomy[Title/Abstract]) OR (Cystectomies[Title/Abstract])) AND (((Hernia[Title/Abstract]) OR (Hernias[Title/ Abstract])) OR (Enterocele[Title/Abstract])) 157 records

\section{Embase and the Cochrane Library through OVID}

\#1 (hernia or hernias or enterocele).kw. 19241

\#2 (cystectomy or cystectomies).kw. 4697

\#3 1 and 230 records

\section{Web of Science}

\#1 TOPIC: (Hernia) OR TOPIC: (Hernias) OR TOPIC: (Enterocele) 95616

\#2 TOPIC: (Cystectomy) OR TOPIC: (Cystectomies) 26667

\#3 1 and 2276 records 
Table S1 The Egger's test and Begg's test of outcomes included in this meta-analysis

\begin{tabular}{lcc}
\hline Outcomes & Egger's test & Begg's test \\
\hline Overall radiographic PH incidence & 0.272 & 0.386 \\
Clinically evident PH incidence & 0.365 & 0.175 \\
1-yr radiographic PH incidence & 0.221 & 0.221 \\
2-yr radiographic PH incidence & 0.695 & 0.452 \\
Overall symptom-related incidence & 0.649 & 0.707 \\
Surgical repair & 0.032 & 0.474 \\
Recurrence of repairs & 0.137 & 0.462 \\
\hline
\end{tabular}

$\mathrm{PH}$, parastomal hernia. 

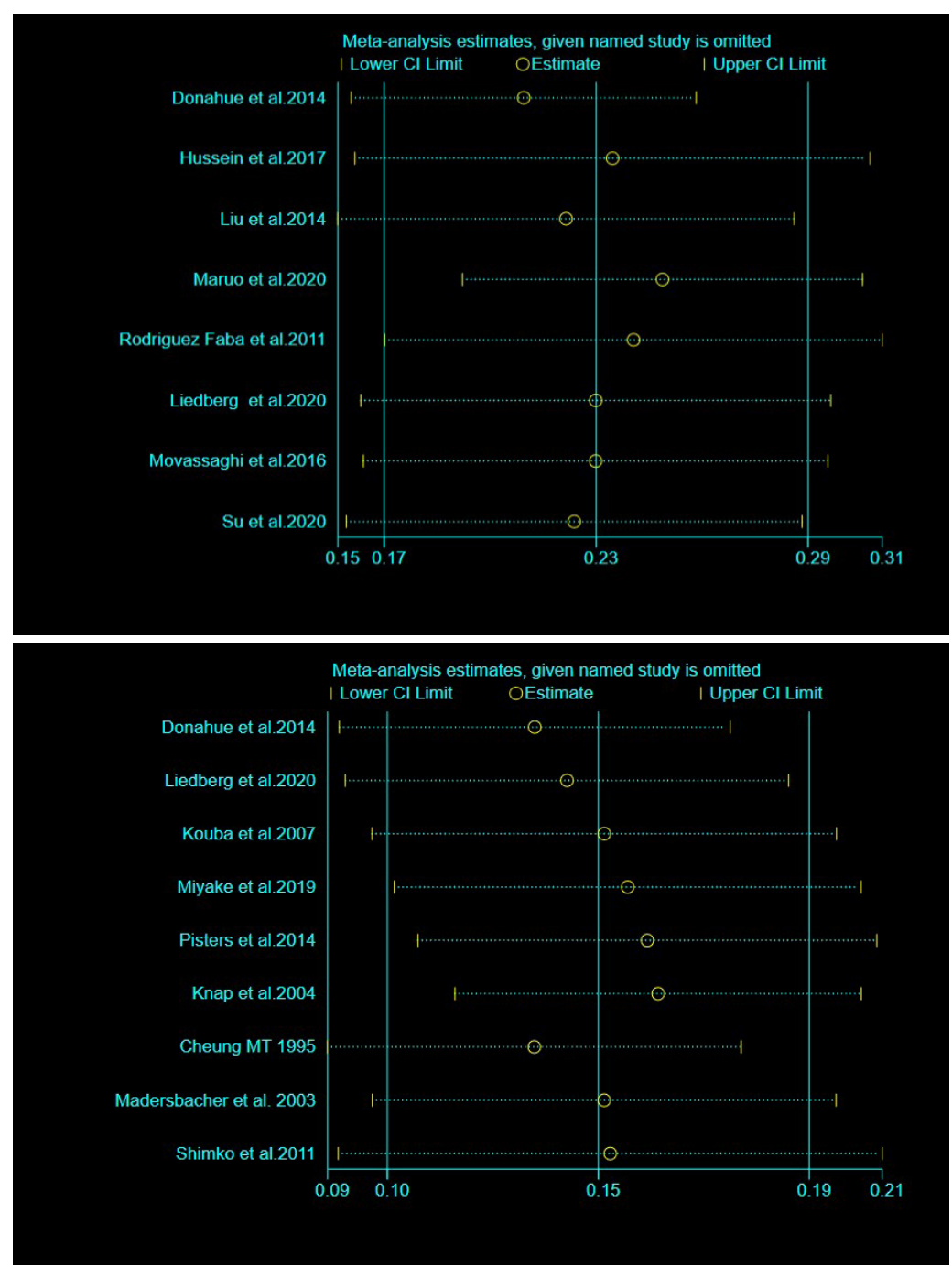

Figure S1 Overall radiographic PH incidence (A); clinically evident PH (B). PH, parastomal hernia. 